\title{
Arborescences
}

Revue d'études françaises

\section{Normes objectives et variation socio-stylistique : le français québécois parlé en contexte d'entrevues télévisées}

\section{Anne-José Villeneuve}

Numéro 7, décembre 2017

La norme orale en français laurentien

URI : https://id.erudit.org/iderudit/1050968ar

DOI : https://doi.org/10.7202/1050968ar

Aller au sommaire du numéro

\section{Éditeur(s)}

Département d'études françaises, Université de Toronto

ISSN

1925-5357 (numérique)

Découvrir la revue

Citer cet article

Villeneuve, A.-J. (2017). Normes objectives et variation socio-stylistique :

le français québécois parlé en contexte d'entrevues télévisées. Arborescences, (7), 49-66. https://doi.org/10.7202/1050968ar

\section{Résumé de l'article}

Les variétés linguistiques nord-américaines se distinguent depuis longtemps de leurs contreparties européennes. Pourtant, les « français d'ici » demeurent stigmatisés puisque souvent associés systématiquement au parler des classes populaires ou à des vernaculaires jugés trop éloignés de la norme fantasmée associée à l'écrit. Or, l'usage québécois oral n'est pas exempt de styles soutenus, dont l'essentiel reste à décrire : plusieurs études ont porté sur le vernaculaire, mais seules quelques-unes se sont penchées sur les usages spontanés en situations plus formelles. Le présent article propose la description d'une norme objective en décrivant l'usage linguistique des élites culturelles et politiques du Québec dans deux contextes d'entrevues télévisées sur les ondes de Radio-Canada. En plus d'étoffer les descriptions existantes du français québécois parlé, la comparaison des deux contextes permet de mesurer la variation stylistique chez les individus. Les résultats de l'analyse de deux variables susceptibles de distinguer l'oral soutenu de l'écrit-l'alternance des auxiliaires et la négation verbale-montrent que les styles observées en contexte d'entrevues télévisées se situent à mi-chemin entre les vernaculaires et le 'standard' écrit. En examinant d'une part des normes objectives émanant d'un groupe socio-culturellement valorisé et, d'autre part, la variation socio-stylistique en français québécois du $21^{\mathrm{e}}$ siècle, la présente contribution éclaire la sociolinguistique française et ses applications pédagogiques éventuelles en contexte canadien.
Tous droits réservés @ C Département d'études françaises, Université de Toronto, 2018
Ce document est protégé par la loi sur le droit d'auteur. L'utilisation des services d'Érudit (y compris la reproduction) est assujettie à sa politique d'utilisation que vous pouvez consulter en ligne.

https://apropos.erudit.org/fr/usagers/politique-dutilisation/ 


\section{SOMMAIRE}

1 Marie-Hélène Côté, Université de Lausanne

Anne-José Villeneuve, Université de l'Alberta Introduction

17 Davy Bigot, Université Concordia

Regard rétrospectif sur la norme du français québécois oral

33 Marie-Hélène Côté, Université de Lausanne

Wim Remysen, Université de Sherbrooke

Le "bon usage du français au Québec» selon le Multidictionnaire de la langue française: le cas de la prononciation

49 Anne-José Villeneuve, Université de l'Alberta

Normes objectives et variation socio-stylistique: le français québécois parlé en contexte d'entrevues télévisées

67 Monelle Guertin, Université du Québec à Montréal

Variation sociophonétique dialectale et stylistique: quelle est la langue cible en français langue seconde à Montréal?

90 Suzie Beaulieu, Université Laval

Leif French, Sam Houston State University

Samuel Gagnon, Université Laval

I'veulent-tu parler comme nous-autres? Opinions d'apprenants sur la forme interrogative totale en français laurentien 


\title{
Normes objectives et variation socio-stylistique: le français québécois parlé en contexte d'entrevues télévisées
}

\author{
Anne-José Villeneuve, Université de l'Alberta
}

\section{Résumé}

Les variétés linguistiques nord-américaines se distinguent depuis longtemps de leurs contreparties européennes. Pourtant, les "français d'ici» demeurent stigmatisés puisque souvent associés systématiquement au parler des classes populaires ou à des vernaculaires jugés trop éloignés de la norme fantasmée associée à l'écrit. Or, l'usage québécois oral n'est pas exempt de styles soutenus, dont l'essentiel reste à décrire: plusieurs études ont porté sur le vernaculaire, mais seules quelques-unes se sont penchées sur les usages spontanés en situations plus formelles. Le présent article propose la description d'une norme objective en décrivant l'usage linguistique des élites culturelles et politiques du Québec dans deux contextes d'entrevues télévisées sur les ondes de Radio-Canada. En plus d'étoffer les descriptions existantes du français québécois parlé, la comparaison des deux contextes permet de mesurer la variation stylistique chez les individus. Les résultats de l'analyse de deux variables susceptibles de distinguer l'oral soutenu de l'écrit - l'alternance des auxiliaires et la négation verbale montrent que les styles observées en contexte d'entrevues télévisées se situent à mi-chemin entre les vernaculaires et le 'standard' écrit. En examinant d'une part des normes objectives émanant d'un groupe socio-culturellement valorisé et, d'autre part, la variation socio-stylistique en français québécois du $\mathrm{XxI}^{\mathrm{e}}$ siècle, la présente contribution éclaire la sociolinguistique française et ses applications pédagogiques éventuelles en contexte canadien.

\section{Introduction}

Des études ont démontré que les variétés d'anglais, d'espagnol et de portugais parlées en Amérique présentent des caractéristiques lexicales, phonologiques et morphosyntaxiques qui les distinguent de leurs contreparties européennes. Il n'est donc pas étonnant que certains usages laurentiens (par exemple, l'emploi fréquent du marqueur de discours là ou l'ordre des clitiques pronominaux dans des impératives telles que dis-moi-le) diffèrent eux aussi de ceux des Français. Or, bien que solidement établis au Québec, certaines de ces formes sont stigmatisées dans les discours publics et dans l'enseignement, car on les associe systématiquement à des usages familiers jugés trop éloignés d'un certain «français standard». Cette conception d'une norme monolithique, qui est souvent basée sur le français écrit, mais qu'on croit parlée couramment en France, continue d'habiter l'imaginaire francophone. Elle renforce le mythe d'une norme fantasmée, qui n'a "parfois qu'une faible zone d'adhérence avec le réel» (Moreau 1997). En effet, combien de fois a-t-on entendu remise en question l'existence même d'un français québécois oral soutenu?

L'idéologie d'un «standard» monolythique ou d'une norme fantasmée repose en grande partie sur une sous-estimation des distinctions entre langue orale et langue écrite (ou variation diamésique) : «la norme est liée à l'écrit, puisque c'est pour l'essentiel là qu' est intervenue et que n'en finit pas d'intervenir 
la standardisation" (Gadet 1995: 20). Dans notre quête d'une meilleure description de normes objectives orales, il est donc pertinent de se demander dans quelle mesure certains styles oraux en français québécois reflètent effectivement l'écrit par une plus grande présence de formes morphosyntaxiques explicitement prescrites à l'écrit (p. ex., la particule négative ne) ou par le maintien de segments reflétés orthographiquement (p. ex., /1/ final dans les clitiques sujets ou les groupes obstruente-liquide). Ce faisant, nous testons indirectement la validité d'un modèle mixte de la variation en français (Villeneuve et Auger 2013) qui reconnaît le caractère intrinsèquement variable du français parlé spontanément tout en admettant que certaines formes appartenant avant tout au mode écrit puissent apparaittre dans des situations hautement formelles en transcendant la frontière diglossique entre l'oral spontané et l'écrit, comme le montre la Figure 1.

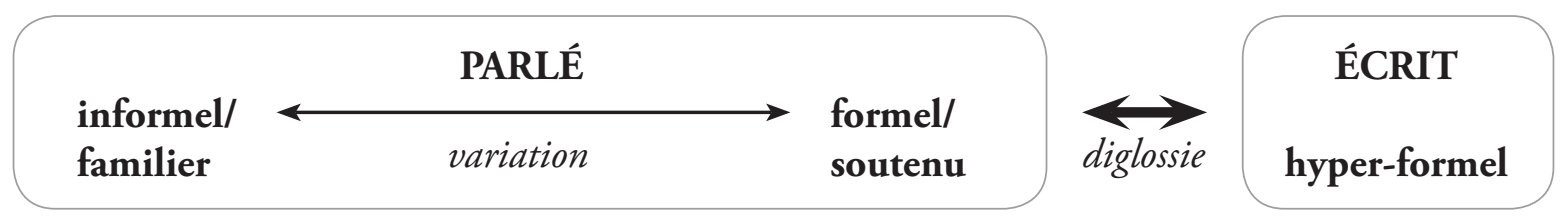

Figure 1: Modèle variationniste mixte du français (adapté de Villeneuve et Auger 2013: 127)

Le présent article présente un nouveau corpus de français québécois (désormais FQ) plus soutenu visant à illustrer une norme objective basée sur l'usage réel de locuteurs contemporains appartenant aux élites culturelles et politiques du Québec. En analysant ce corpus par le biais de méthodes quantitatives issues de la sociolinguistique variationniste, nous cherchons à combler une lacune dans la littérature, c'est-à-dire à mesurer l'effet que jouent les facteurs sociaux et stylistiques (ou socio-situationnels) dans les styles plus soutenus du FQ d'aujourd'hui. Nous abordons d'abord la notion de variation stylistique dans le contexte québécois (section 2), puis nous décrivons le corpus On prend toujours un train et l'intérêt qu'il présente pour l'étude des styles plus soutenus en FQ (section 3). Une illustration de la variation stylistique par l'analyse de l'alternance des auxiliaires et de la négation verbale, deux variables susceptibles de distinguer l'oral soutenu de l'écrit, est présentée dans la section 4. Quelques remarques sur les applications pédagogiques de cette recherche sont offertes en guise de conclusion.

\section{Normes objectives et vernaculaire(s) laurentien(s)}

La variation est inhérente à la langue et se manifeste notamment dans les domaines morphosyntaxique et phonique: il existe en effet différentes façons d'exprimer le futur (p. ex., je vais partir demain ou je partirai demain) ou de prononcer une phrase comme ils ont une grande table (p. ex., liaison entre ils et ont, réalisation du /1/ final de ils ou de table). En observant la langue dans la société et en encodant divers aspects du contexte dans lequel elle se produit, les sociolinguistes ont démontré que la variation dans la langue spontanée n'est pas aléatoire mais respecte des patrons systématiques pouvant être prédits par des modèles probabilistes (Cedergren et Sankoff 1974, Labov 1972, Tagliamonte 2006). En fait, les analyses menées à l'aide des méthodes quantitatives de la sociolinguistique variationniste illustrent bien la façon dont les facteurs sociaux (sexe, âge, profession, etc.), stylistiques (formalité de la situation de communication, thèmes abordés, etc.) et linguistiques (catégorie du mot, contexte syllabique, etc.) influent sur la langue.

Alors que les vernaculaires continuent d'être documentés et décrits, l'usage soutenu du FQ demeure relativement peu étudié en sociolinguistique. Nous proposons donc de combler cette lacune 
en produisant une description détaillée du français parlé couramment au $\mathrm{xxI}^{\mathrm{e}}$ siècle dans le but de déterminer s'il existe un FQ oral soutenu dont la morphosyntaxe serait distincte tant du vernaculaire que du français écrit. Plus précisément, le présent article cherche à répondre aux questions de recherche suivantes: 1. Quelles sont les caractéristiques qui distinguent le FQplus soutenu, tel que le parlent les élites culturelles et politiques en contexte d'entrevue télévisée? 2. Quels facteurs sociaux et situationnels influent sur la réalisation d'une forme linguistique plutôt que d'une autre?

\subsection{Entre vernaculaire(s) et «standard»: la variation socio-stylistique}

La notion de style est centrale à la sociolinguistique puisqu'elle permet de rendre compte qu'il existe, en plus de la variation sociale entre les pratiques de divers groupes de locuteurs (ou variation diastratique), des différences stylistiques au sein du parler d'un locuteur donné (ou variation diaphasique). Même si on s'entend pour dire que l'ampleur de la variation stylistique est généralement moindre que celle de la variation sociale (Bell 1984), la façon de concevoir le style varie selon les approches. D'une part, pour Labov (1972), un locuteur emploiera tantôt un style familier («casual»), tantôt soutenu («careful») selon le degré d'attention qu'il porte à son langage, le vernaculaire représentant le style le moins surveillé de tous. Selon cette approche, le parler varierait dans divers styles dans la mesure où le locuteur reproduit, plus ou moins consciemment, les variations sociales qu'il perçoit au sein des diverses classes socio-économiques de sa communauté linguistique; le stylistique serait donc en quelque sorte un reflet du social. Dans ses travaux sur diverses variétés d'anglais américain, Labov distingue ainsi le familier du soutenu en s'appuyant sur des critères tels que le type de discours (p. ex., récit personnel ou explication) ou le thème abordé (p. ex., souvenirs de jeux d'enfants ou prise de position sur l'éducation de ceux-ci). D'autre part, Bell $(1984,2001)$ accorde une plus grande importance à l'interlocuteur, réel ou imaginé, et à la situation de communication dans la variation stylistique. Sa proposition du concept d'accommodation langagière ("audience design»), inspiré des travaux de H. Giles, tient compte du fait que les choix du locuteur s'opèrent dans le cadre d'un acte de communication au cours duquel celui-ci entre en relation avec son interlocuteur dans un contexte donné. D'autres approches encore, qui accordent une plus grande part d'agentivité au locuteur (p. ex., Eckert 2000), entrevoient la variation stylistique comme reflétant des choix de formes parmi un éventail de ressources, entre autres linguistiques, dont dispose chaque locuteur. Comme les styles vestimentaires, les styles oraux représentent ainsi un amalgame d'éléments (vêtements ou variables) se combinant pour projeter une identité.

Un grand manque de connaissance persiste toutefois en ce qui a trait à la variation stylistique en FQ. En effet, tandis que les travaux en sociolinguistique variationniste ont amélioré notre compréhension de la structure du vernaculaire parlé dans des contextes familiers, l'usage soutenu demeure relativement inexploré. Au cours des 50 dernières années, des équipes de chercheurs ont développé des modèles linguistiques basés sur de grands corpus oraux recueillis par le biais d'entrevues semi-dirigées (Labov 1984) enregistrées en milieu francophone majoritaire ${ }^{1}$ : à Montréal (Sankoff et al. 1976, Thibault et Vincent 1990, Vincent et al. 1995), à Ottawa-Hull (Poplack 1989), à Québec (Deshaies 1981), au Saguenay (Paradis 1985), etc. Grâce aux enquêtes faites à Montréal en 1971, 1984 et 1995 , le FQ est d'ailleurs souvent cité en exemple pour illustrer des principes théoriques sur la variation et le changement linguistiques, tant à l'échelle de la communauté que chez l'individu (Blondeau 2001,

1. Mis à part les travaux de R. Mougeon et de Beniak (1989, 1991, entre autres), ce n'est que plus tard que l'on s'est intéressé systématiquement aux usages vernaculaires laurentiens des francophones vivant en situation minoritaire en Ontario ou dans l'Ouest canadien. 
2006, Blondeau et al. 2002, Sankoff et Blondeau 2007). Malheureusement, on ne dispose toujours pas de description empirique rigoureuse des usages québécois en situations plus formelles.

Motivés par le doute qui planait quant à l'existence même d'une norme dominante ou d'un «standard» en FQ (Barbaud 1998), des chercheurs ont développé des descriptions du lexique québécois «standard" (Cajolet-Laganière et Martel 1995, Martel et Cajolet-Laganière 2000). La publication d'Usito, un dictionnaire dynamique en ligne, est née de cette quête d'une norme québécoise en constante évolution. Un problème subsiste toutefois: le lexique se prête difficilement aux analyses quantitatives, malgré quelques études du genre (Beeching 2012). Certains ont donc cherché des normes objectives pour la phonologie et la morphosyntaxe soutenues dans les corpus médiatiques et chez des locuteurs représentant une certaine élite culturelle, politique ou intellectuelle.

En tant que diffuseur public francophone, Radio-Canada est largement reconnue comme un modèle de la norme d'ici, du français «standard" parlé au Canada (Radio-Canada 2003). De façon générale, les Canadiens voient cette norme dans le parler des lecteurs de nouvelles, journalistes et autres professionnels de la société d'état (Bouchard et Maurais 2001, Gendron 1990). Les études de Cox (1998) et de Reinke (2005) ont déjà bien documenté la prononciation des lectrices et lecteurs de nouvelles, mais si cette description constitue une norme objective pertinente sur le plan phonique, elle en dit peu sur la morphosyntaxe de l'oral spontané; les textes préparés d'avance en prévision du bulletin et lus en direct à l'antenne demeurent de l'écrit oralisé. Les applications pédagogiques d'un tel corpus sont ainsi très limitées: bien qu'il puisse aider la compréhension de l'oral très soutenu, le style des lecteurs de nouvelles implique un niveau de formalité trop élevé pour servir de modèle au développement d'une bonne conscience sociolinguistique (p. ex., variation stylistique) transférable aux conversations courantes.

\subsection{L'entrevue télévisée: représentation d'une norme orale objective}

Les corpus télévisuels spontanés offrent la promesse d'une description empirique d'un style situé à mi-chemin entre les vernaculaires et la réalisation orale d'un «standard" écrit, ce que démontrent les travaux de Reinke (2005) et de Bigot $(2008,2011)$. Dans l'analyse de Reinke, certaines entrevues de Radio-Canada montraient déjà une plus grande proportion de formes courantes: "plus le degré de formalité de l'émission est élevé, moins le taux de variantes familières l'est» (p. 25). Elle a notamment constaté que les entretiens au sein des émissions d'intérêt général (ou de «Type 2 », p. 16) comportaient davantage de variantes familières que les émissions d'information (Type 1) mais moins que les émissions ludiques (Type 3). Par exemple, si l'on se limite à la variation morphosyntaxique, l'emploi de la particule négative ne passait de 79,8\% dans les émissions d'information à 35,7\% dans les émissions d'intérêt général, puis à $21,2 \%$ dans les émissions ludiques. Elle soutient, contrairement à Ashby (1984), que l'effacement de ne n'est pas nécessairement un phénomène plus courant au Québec, mais est une "question de style». À partir de son analyse de diverses variables dépendantes, dont la plupart relèvent de la phonologie ou de la phonétique (p. ex., effacement du /1/ ou du schwa dans les clitiques pronominaux sujets, simplification des groupes consonantiques), Reinke (2005: 26) conclut que la variation à la télévision québécoise «reflète la diversité sociale et linguistique».

À peu près au même moment, Bigot $(2008,2011)$ analysait des entrevues enregistrées dans le cadre de l'émission d'information Téléjournal Le Point (désormais Le Point), un corpus comparable aux émissions d'information (Type 1) de Reinke et dont on estime qu'il représente le discours des élites culturelles québécoises (Barbaud 1998) — des universitaires, politiciens, artistes, dirigeants 
d'entreprises - en situation plus formelle. À la lumière de son analyse statistique de diverses formes familières (p. ex., concordance négative avec personne ou rien, /1/ non étymologique après ça, réalisation $\mathrm{du} / \mathrm{t} /$ final dans le participe passé fait), Bigot conclut que la norme objective du français parlé au Québec se conforme en grande partie aux règles décrites par les grammairiens (Grevisse et Goosse 1993). Il constate également que, pour plusieurs variables dépendantes, les femmes et les locuteurs plus âgés emploient davantage de formes normatives que leurs homologues masculins et plus jeunes, comme le prédisent deux principes classiques de la sociolinguistique variationniste.

Il subsiste néanmoins un manque de connaissance sur la variation socio-stylistique dans l'usage plus soutenu étant donné que les méthodes statistiques employées auparavant - les fréquences relatives chez Reinke, les tests de chi-carré chez Bigot - mesurent l'effet absolu des facteurs sociaux et linguistiques, mais pas leur effet relatif. Les données brutes des entrevues Le Point n'étant plus accessibles (Bigot, c.p.), il est impossible de les soumettre à des analyses à régression multiple ou à des tests de corrélation. Ainsi, malgré la promesse que représente le genre de l'entrevue télévisée pour la description d'une norme objective au Québec, plusieurs questions demeurent sans réponse (Bigot et Papen 2013).

\section{Le corpus On prend toujours un train}

Pour aborder des questions liées au FQ plus soutenu, nous examinons une nouvelle série d'entrevues télévisées sur les ondes de Radio-Canada entre 2008 et 2013 dans le cadre de l'émission On prend toujours un train ${ }^{2}$ (désormais Un Train), animée et co-produite par Josélito Michaud. Ayant acquis quelques-unes des entrevues Le Téléjournal Le Point (Bigot 2008), nous comparons les deux corpus pour les quelques locuteurs ayant participé aux deux émissions télévisées. Les entrevues de Un Train durent de 13 à 25 minutes chacune; les entrevues de Le Point, de 8 à 10 minutes.

Puisque chaque émission est susceptible de faire ressortir un style légèrement différent chez les locuteurs interviewés, la comparaison des deux corpus permet l'étude de la variation stylistique. Axées sur la vie personnelle et sur les émotions (deuil, maladie grave, événement tragique), les entrevues enregistrées dans le cadre de Un Train représentent un style légèrement plus soutenu que le vernaculaire, mais tout de même familier. D'une part, elles se rapprochent de l'entrevue semi-dirigée où, pour réduire l'attention que le locuteur porte à son parler, le sociolinguiste lui demande de raconter divers événements forts en émotions ou de parler de son enfance (Labov 2001). D'autre part, le caractère public de l'entrevue télévisée lui confère une formalité supplémentaire. Quant au corpus Le Point, nous estimons, comme d'autres avant nous (Barbaud 1998, Bigot 2008), qu'il se rapproche davantage du pôle "standard» ou d'un style plus soutenu ou contrôlé, tout en demeurant de l'oral spontané. Non seulement l'accent y est-il mis sur une posture et des thèmes professionnels, mais les intervieweurs sont également des journalistes de Radio-Canada, perçus par bon nombre de Canadiens comme des modèles langagiers. Par ailleurs, les formes d'adresse employées par les intervieweurs sont un indice supplémentaire de la différence de formalité entre les deux corpus. Dans le corpus principal Un Train, l'intervieweur emploie le tutoiement ou le vouvoiement avec ses invités, en fonction de divers facteurs sociaux (p. ex., proximité sociale antérieure à l'entrevue), alors que tous les invités sont vouvoyés dans le corpus complémentaire Le Point.

2. D'une grande popularité auprès du public, l'émission a également reçu les éloges de la critique par le biais de nominations aux Prix Gémeaux de l'Académie canadienne du cinéma et de la télévision en 2010 et en 2013. 
Tableau 1: Corpus On prend toujours un train (2008-2011)

\begin{tabular}{|l|c|c|}
\hline \multicolumn{1}{|c|}{ Sexe } & Pré-Révolution tranquille & Post-Révolution tranquille \\
\hline Femmes & 8 & 8 \\
Hommes & 8 & 8 \\
\hline
\end{tabular}

Comme le démontre le Tableau 1, les locuteurs sélectionnés pour le corpus Un Train sont répartis également entre les sexes et deux groupes d'âge: les adultes nés avant 1960 et ceux nés entre 1960 et l'adoption au Québec de la Loi sur la langue officielle, ou Loi 22, en juillet 1974 (Bourhis et Landry $2002)^{3}$. Si l'on considère une entrée à l'école à 6 ans, cela signifie que le plus jeune groupe a accédé au système scolaire au début de la Révolution tranquille alors que la scolarisation du groupe d'adultes plus mûrs s'est faite avant ce point tournant dans l'histoire du Québec. La Révolution tranquille a été choisie comme date charnière en raison de son impact sur la conception de la langue et sur les discours normatifs des chroniqueurs linguistiques (Remysen 2011); elle permet de tester l'effet potentiel d'un changement culturel collectif sur les comportements linguistiques. Les autres variables sociales indépendantes dont nous mesurons l'effet sur l'usage linguistique incluent la catégorie professionnelle du locuteur (artistes, politiciens, animateurs, etc.), adaptée de la classification de Bigot (2011), et son origine régionale (région de Montréal, de Québec ou autre).

Les entrevues ont été transcrites selon un protocole adapté de Bachy et al. (2004/2007), qui reflète plusieurs des propriétés de l'oral tout en employant une orthographe conventionnelle facilitant la lecture (cf. 1, extrait de l'entrevue de Gilles P., lignes 98-104).

(1) L0 avez-vous l'impression avec un recul que c’était le= c’était ça qu'y avait à faire?

L1 ben j'avais une carrière et c'est c'que je menais je ne me voyais pas dans d'autres disciplines où j'aurais eu un travail plus sédentaire pis dire bon "j'vais cajoler mon fils pis euh j'vais le faire connaître une croissance euh semblable à tout le voisinage" euh y avait ça d'une part y a aussi le fait que même si j'étais peut-être resté avec euh ma femme ça aurait été qu'une échéance qui aurait été remise euh quant à l'éclatement de = de l'union et mon fils serait euh tombé dans $l==$ dans l'même pattern si on veut

Les tours de parole de l'intervieweur, Josélito Michaud, sont identifiés par «L0»; ceux des interlocuteurs, par «L1». Les marqueurs discursifs (ben, bon, là, etc.), les hésitations (euh, euhm, etc.) et les faux départs sont notés tels quels et les éléments syntaxiques dont l'omission est prévisible (p. ex., $i l$ impersonnel, particule négative ne) ne sont pas restaurés ou "corrigés", ce qui permet de refléter plus fidèlement l'oral spontané. Par contre, mise à part la présence variable du schwa dans les monosyllabes

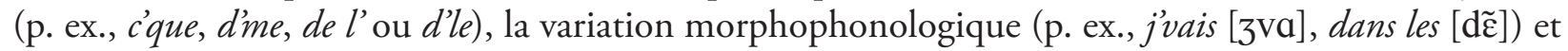
les réductions de nature phonique (p. ex., simplification de groupes consonantiques, effacement du /1/ dans les clitiques) ne sont pas représentées comme on le fait dans certaines œuvres théâtrales ou bandes dessinées. En plus d'éviter d'alourdir la lisibilité des transcriptions, ce choix méthodologique facilite la recherche automatisée des variables dépendantes. Les emprunts lexicaux à l'anglais sont mis en évidence par le biais de l'italique (pattern, party, show, etc.), mais les calques syntaxiques ou sémantiques ne sont

3. Tous les locuteurs du corpus précèdent donc la génération Passe-Partout. Cette émission produite par le ministère de l'Éducation et destinée à l'éducation préscolaire (Hamel 1995, Lemelin 1979) a été marquante pour bon nombre de Québécois nés entre le milieu des années 70 et la fin des années 80, en favorisant entre autres un éveil linguistique stimulé par le personnage de Passe-Montagne. 
pas notés typographiquement. Enfin, les divers morphèmes des mots complexes (parce-que, par-contre, etc.) sont liés par des traits d'union afin de les distinguer de constructions syntaxiques semblables (p. ex., en-fait mon fils en fait encore partie) et de refléter le fait qu'ils ne puissent être décomposés. Ainsi transcrit, le corpus d'entrevues peut facilement être exploité en contexte pédagogique pour aborder des questions liées à l'oral spontané, en comparant par exemple celui-ci à l'écrit soutenu.

Les variables dépendantes à l'étude dans cette recherche ont été choisies en fonction des descriptions antérieures de l'usage québécois soutenu (Bigot 2008, 2011, Bigot et Papen 2013, Ostiguy et Tousignant 2008, Reinke 2005): des variables morphosyntaxiques et phonologiques dont on se demande toujours si elles sont effectivement employées dans des situations plus formelles. Par exemple, Bigot et Papen (2013) soutiennent que la norme n'est pas encore définie en ce qui a trait à l'effacement $\mathrm{du} / 1 /$ des clitiques pronominaux sujets comme il(s) et elle(s), qu'on observe également en français hexagonal (Ashby 1984, François 1974). Dans la section suivante, nous présentons l'analyse de deux variables binaires dont la variante dite "soutenue» coïncide avec l'usage écrit; elles sont donc susceptibles de nous permettre d'évaluer si les normes objectives en FQ oral soutenu correspondent au «standard» écrit.

\section{Décrire l'oral soutenu en français québécois spontané}

Pour déterminer si la production orale en contexte d'entrevue se distingue bel et bien du FQ vernaculaire en se rapprochant de l'écrit, nous présentons ici, pour un sous-ensemble de 24 locuteurs du corpus Un Train (12 hommes, 12 femmes), l'analyse de deux variables morphosyntaxiques binaires dont on estime généralement que les variantes dites "familières" et "soutenues" reflètent la distinction entre oral et écrit: l'alternance des auxiliaires et la négation verbale. Afin de mesurer la variation stylistique au sein même de l'oral plus soutenu, nous comparons également les données de Un Train à celles recueillies dans le cadre de l'émission Le Point pour cinq locuteurs ayant été interviewés dans les deux contextes.

D'une part, nous formulons l'hypothèse que l'emploi de l'auxiliaire avoir avec les verbes pronominaux et de mouvement s'éloignera du vernaculaire en raison de la stigmatisation de cet auxiliaire dans certaines communautés francophones; nous nous attendons donc à une absence de distinction entre oralité et norme écrite pour cette variable. D'autre part, nous croyons que la réalisation variable de la particule négative ne (p. ex., il ne comprend pas) reflètera une distinction claire entre oralité et norme écrite. En effet, plusieurs travaux ont démontré que, bien qu'elle soit employée à l'écrit soutenu ${ }^{4}$, la particule négative est généralement absente du français parlé au Canada, et ce, depuis au moins le milieu du XIx ${ }^{\mathrm{e}}$ siècle (Poplack et St-Amand 2007, Sankoff et Vincent 1977). Les travaux de Ashby (2001) ont confirmé un changement semblable vers la chute graduelle du $\boldsymbol{n} \boldsymbol{e}$ en France entre 1971 et 1995. Nous nous attendons donc à ce que la particule n'apparaissent que rarement dans les deux corpus de FQ oral soutenu.

4. Les travaux portant sur les lettres (Dubois 2011, Martineau 2005) et les textos (Blondeau et al. 2014) montrent que ces genres écrits se rapprochent davantage de la langue orale. 


\subsection{L'auxiliaire avoir avec les verbes de mouvement et les verbes pronominaux: le poids du «standard» écrit?}

Contrairement au standard écrit, où l'emploi de l'auxiliaire être est prescrit avec les verbes pronominaux (cf. 2) et avec un ensemble de verbes inaccusatifs dénotant le mouvement (cf. 3 et 4), on note depuis longtemps de la variation dans le choix de l'auxiliaire dans les variétés parlées à travers la francophonie (Bauche 1920/1946, Conwell et Juilland 1963, Frei 1929/1971, Gesner 1978, Guillot 1932, Russo et Roberts 1999).

(2) parce-que on s'était mariés pour avoir une grosse famille

(3) j’pense que j'ai tombé au tapis dernièrement

(Un Train, Andrée R., 70)

(4) quand il a ouvert la porte le chien est parti sur le lac

(Un Train, Éric L., 220)

(Un Train, Sylvie F., 62)

Dans le contexte canadien, il existe bon nombre d'études sur l'alternance des auxiliaires, tant dans des variétés parlées à Montréal (Sankoff et Thibault 1977) ou dans la région d'Ottawa-Hull (Willis 2000) que dans diverses communautés francophones en milieu minoritaire. Or, si l'emploi d'avoir paraît généralisé en français acadien (King et Nadasdi 2000, Péronnet 1991), même pour les verbes pronominaux, il tend à dépendre de facteurs linguistiques et sociaux en FQ. Léard (1995) note par exemple que l'usage québécois privilégie fortement la variante normative pour certains verbes (p. ex., naître, mourir, aller, (re)venir), mais la variante vernaculaire pour d'autres (p. ex., monter, tomber, passer). Cette différence s'explique en partie par le fait que la seconde catégorie de verbes permet un usage adjectival (p. ex., il est sorti en ce moment, où l'interprétation passée est exclue), car "plus le participe passé peut servir d'adjectif pour exprimer un état ([- complété]), plus il est possible de le conjuguer avec avoir (usage [+ complété])»(Sankoff et Thibault 1977: 99). Socialement, l'emploi d'avoir semble stigmatisé et tend à diminuer avec l'augmentation du niveau d'éducation (voir Hallion Bres 2004 pour le Manitoba), ce qui pourrait avoir favorisé un changement linguistique vers la standardisation de l'usage en 1995, l'emploi d'avoir à Montréal se limitait déjà à 20 \% (Sankoff et al. 2004).

\subsubsection{L'alternance des auxiliaires en français québécois soutenu}

Nous nous sommes donc demandé ce qu'il advient de l'alternance des auxiliaires dans un corpus d'entrevues orales individuelles enregistrées dans les années 2000. Peut-on s'attendre à ce que le changement attesté à Montréal entre 1971 et 1995 soit complété plus de dix ans plus tard? Observera-t-on un usage plus fréquent ou même catégorique d'être dans un corpus d'entrevues individuelles avec des personnalités publiques qu'on associe au «français québécois standard» ? Si cette variable est sujette à la variation socio-stylistique, trouverons-nous une différence significative entre les deux contextes pour la cohorte de cinq locuteurs enregistrés dans les deux contextes? Cette différence impliquera-t-elle un emploi systématiquement moins fréquent d'avoir pendant l'émission Le Point? Nos hypothèses de départ répondent de façon affirmative à chacune de ces questions.

Pour tester nos hypothèses, nous avons extrait toutes les occurrences d'un verbe conjugué à un temps composé où l'alternance est possible en français laurentien ; étant donné l'emploi d'avoir avec les verbes pronominaux dans les français de l'Ouest (Hallion Bres 2006) et dans un corpus montréalais plus récent (Rea 2015), ce contexte est inclus dans la présente analyse. Les cas où il est question de l'état résultant de l'action et ceux où le participe passé est employé de façon adjectivale sont exclus de l'analyse. En plus de considérer l'effet des facteurs sociaux (voir section 3), nous mesurons la contribution 
de facteurs linguistiques tels que le type de contexte (pronominal ou non-pronominal) et le verbe luimême (aller, monter, tomber, passer, etc.), suivant l'approche de Sankoff et Thibault (1980).

L'analyse de 448 occurrences de la variable dans le corpus Un Train confirme notre hypothèse: l'emploi d'avoir est quasi-inexistant dans ce contexte d'entrevue. L'emploi catégorique d'être avec les verbes pronominaux $(\mathrm{N}=142)$, comme dans le vernaculaire, et quasi-catégorique ailleurs $(\mathrm{N}=306)$ semble indiquer que nous nous trouvons ici dans un style oral certes spontané, mais plus soutenu, qui coïncide avec la norme de l'écrit soutenu. Par contre, il existe une légère variation individuelle: seuls deux locuteurs, Éric L. et Dan B., deux chanteurs populaires, font usage d'avoir au cours de leur entrevue à On prend toujours un train. En adoptant une approche identitaire du style (p. ex., Eckert 2000, Coupland 2007), nous pourrions croire que cet emploi de la variante familière est lié à l'image que projettent ces artistes auprès du public québécois. Nous verrons cependant que ce n'est qu'une partie de la réponse.

\subsubsection{L'alternance des auxiliaires et la variation stylistique}

Comparons maintenant les données issues des deux corpus. Contrairement à notre hypothèse, l'usage d'avoir avec les verbes non-pronominaux dans l'entrevue Le Téléjournal Le Point augmente chez trois des locuteurs de la cohorte (voir Tableau 2). Par exemple, Dan B. emploie deux occurrences d'avoir sur un total potentiel de 13 dans Un Train, mais trois sur un total potentiel de 5 dans Le Point. Des valeurs de p supérieures à 0,05 révèlent toutefois que la différence de proportion d'avoir entre les deux corpus n'est pas statistiquement significative chez ces locuteurs. Il semble donc que l'usage d'avoir, déjà quasi inexistant dans le corpus Un Train, ne soit pas véritablement sujet à la variation stylistique, du moins en ce qui a trait à la norme objective représentée dans les entrevues télévisées avec des personnalités publiques québécoises. Il faut toutefois interpréter cette conclusion avec prudence étant donné le peu de données fournies par le corpus Le Point $(\mathrm{N}=18)$.

Tableau 2: Emploi d'avoir dans deux contextes d'entrevue: Un Train et Le Point

\begin{tabular}{|c|c|c|c|c|c|c|}
\hline Catégorie & Locuteurs & $\begin{array}{l}\text { Train } \\
\text { \% avoir }\end{array}$ & $\mathbf{N}$ & $\begin{array}{l}\text { Point } \\
\% \text { avoir }\end{array}$ & $\mathbf{N}$ & $\begin{array}{l}\text { p exact de } \\
\text { Fisher }\end{array}$ \\
\hline \multirow[t]{2}{*}{ Artistes } & Ginette R. & 0,0 & 10 & 0,0 & 4 & 1,000 \\
\hline & Dan B. & 15,4 & 13 & 60,0 & 5 & 0,098 \\
\hline \multirow[t]{3}{*}{ Politiciens } & Liza F. & 0,0 & 13 & 50,0 & 2 & 0,133 \\
\hline & André B. & 0,0 & 4 & - & - & - \\
\hline & Mario D. & 0,0 & 11 & 28,6 & 7 & 0,137 \\
\hline
\end{tabular}

Par ailleurs, passer, le seul verbe avec lequel les femmes emploient avoir, représente à lui seul plus de la moitié des cas d'avoir; il n'est d'ailleurs employé avec être par aucun de ces cinq locuteurs, tant dans le corpus Un Train $(\mathrm{N}=5)$ que dans Le Point $(\mathrm{N}=4)$. Or, selon l'OQLF, bien que "passer s'emploie aujourd'hui le plus souvent avec être, du moins à la forme intransitive", l'auxiliaire avoir n'est pas considéré comme fautif, même dans la langue écrite ; dans ce contexte, il peut toutefois «être senti comme vieilli» (Banque de dépannage linguistique, Office québécois de la langue française [OQLF]). 
(5) a. jpense que j'ai tombé au tapis dernièrement

(Un Train, Éric L., 220)

b. après ça là j'ai passé à moi pis j’ai fait un burnout

(Un Train, Dan B., 290)

c. j'ai connu ça j'ai passé proche d'y rester

d. j'ai passé à travers toutes les phases du deuil

(Un Train, Dan B., 310)

(6) a. quand j'ai parti du Parti libéral

(Un Train, Brigitte P., 98)

b. ça a sorti comme ça

(Le Point, Mario D., 136)

(Le Point, Dan B., 43)

(Le Point, Dan B., 42)

(7) a. tout ça a repassé par moi

b. quatre-vingt-dix pour cent [...] a passé par les arts

(Le Point, Dan B., 140)

c. ça a peut-être passé un peu vite

(Le Point, Liza F., 11)

d. on avait= on a passé à un cheveu d'gagner en Mauricie

(Le Point, Mario D., 106)

On note ainsi que les 21 locuteurs se conformant le plus à la norme écrite n'emploient avoir en contexte non-pronominal qu'avec le verbe passer. Par ailleurs, l'effet lexical observé auparavant (Sankoff et Thibault 1980) se confirme dans notre corpus au-delà du verbe passer, comme l'indique le Tableau $3^{5}$.

Tableau 3: Alternance des auxiliaires dans les corpus Un Train et Le Point, selon les verbes et les locuteurs

\begin{tabular}{|c|c|c|c|c|c|c|c|c|}
\hline \multirow[t]{2}{*}{ Groupes } & \multicolumn{2}{|c|}{$\begin{array}{c}\text { aller, (re)venir, } \\
\text { devenir, arriver, } \\
\text { (r)entrer }\end{array}$} & \multicolumn{2}{|c|}{$\begin{array}{l}\text { (re)partir, retourner, } \\
\text { (re)descendre, } \\
\text { (re)monter }\end{array}$} & \multicolumn{2}{|c|}{$\begin{array}{c}\text { (re)sortir, (re)tomber, } \\
\text { rester }\end{array}$} & \multicolumn{2}{|c|}{ passer } \\
\hline & \% avoir & $\mathbf{N}$ & \% avoir & $\mathbf{N}$ & \% avoir & $\mathbf{N}$ & \% avoir & $\mathbf{N}$ \\
\hline 3 locuteurs & 0,0 & 23 & 14,3 & 7 & 18,1 & 11 & 100,0 & 5 \\
\hline 21 locuteurs & 0,0 & 196 & 0,0 & 46 & 0,0 & 30 & 66,7 & 6 \\
\hline TOTAL & 0,0 & 219 & 1,9 & 53 & 4,9 & 41 & 81,8 & 11 \\
\hline
\end{tabular}

Si l'on se fie aux données de notre corpus d'entrevues télévisées à Radio-Canada, mise à part une faible variation idiolectale distinguant les locuteurs, l'emploi des auxiliaires en FQ soutenu se conforme à l'écrit normatif. Nos résultats semblent d'ailleurs soutenir l'hypothèse d'une standardisation graduelle de l'usage dans l'emploi des auxiliaires, mise de l'avant par Sankoff, Thibault et Wagner (Sankoff et al. 2004), et correspondre à la description de Bigot (2011) selon laquelle la norme québécoise se conforme à l'usage écrit que décrivent les ouvrages normatifs tels que le Bon Usage.

Ayant déterminé que l'usage soutenu se conforme en grande partie à la norme écrite pour l'alternance des auxiliaires, tournons-nous maintenant vers l'expression variable de la particule négative $n e$.

\subsection{La négation verbale: la particule ne comme marqueur de formalité}

La présence variable de la particule négative ne est probablement la variable sociolinguistique par excellence en français contemporain (Coveney 1996/2002), comme l'atteste la multitude d'études portant sur cette variable depuis le milieu du $\mathrm{xx}^{\mathrm{e}}$ siècle (voir Gadet 2000 pour un aperçu de la littérature). Alors que la variante normative, prescrite pour l'écrit soutenu, demeure relativement productive dans les français d'outre-Atlantique, elle est en régression à l'oral depuis au moins le XIX ${ }^{\mathrm{e}}$ siècle (Martineau

5. Étant donnée l'absence de variation stylistique entre les deux corpus, les données de Un Train et de Le Point sont combinées dans le Tableau 3. 
et Mougeon 2003) et elle est essentiellement disparue de l'usage nord-américain. Selon les sociolinguistes, son emploi dans le domaine laurentien, inférieur à $1 \%$, se limite aujourd'hui à une marque stylistique de formalité (Poplack et St-Amand 2009, Sankoff et Vincent 1977).

\subsubsection{La négation verbale en français québécois soutenu}

Nous avons donc voulu déterminer si la particule négative est employée plus fréquemment en contexte d'entrevue télévisée, ce qui pointerait vers un style soutenu se distinguant du vernaculaire décrit dans les corpus de Montréal ou d'Ottawa-Hull, et si les locuteurs la manipulent lorsque la formalité de la situation augmente, comme entre les entrevues Un Train et Le Point.

Comme le veut la tradition sociolinguistique, nous avons recensé toutes les occurrences de la négation verbale et exclu les cas ambigus de liaison en [n] (p. ex., on [n] est pas parfait). Dans la présente étude, nous présentons les résultats d'analyses préliminaires en mettant l'accent sur les facteurs sociaux, notamment la variation individuelle. Ce choix méthodologique permet de dégager l'effet idiolectal provenant de locuteurs dont l'usage particulièrement élevé de la particule négative les distingue du reste du groupe, tout en indiquant la pression normative associée à cette variante.

L'analyse du corpus Un Train révèle un taux d'emploi de ne de près de $8 \%(\mathrm{~N}=1431)$, ce qui est beaucoup plus élevé que dans les corpus de Montréal 1971-1995 et d'Ottawa-Hull. Toutefois, le Tableau 4 montre que quatre expressions fréquentes (p. ex., il fallait pas, y a pas d'doute, c'était pas moi, je sais pas si j'aurais l'courage) se démarquent clairement des autres constructions ( $\mathrm{p} \leq .001)$ par l'absence (quasi-)catégorique de la particule négative. Leur effet fortement défavorable sur l'emploi de la particule ayant été documenté tant dans les variétés canadiennes que dans les variétés européennes (Martineau et Mougeon 2003, Moreau 1986), nous avons exclu ces constructions apparemment lexicalisées ou pragmaticalisées - ou sur le point de l'être - afin d'obtenir une mesure fiable de l'emploi véritablement variable de $n e$.

Tableau 4: Emploi de ne dans les constructions fréquentes, corpus Un Train

\begin{tabular}{|c|c|c|c|c|}
\hline Construction & $\%$ ne & ne & absence & $\mathbf{N}$ \\
\hline (il) FALLOIR & 0,00 & 0 & 12 & 12 \\
\hline (il) y AVOIR & 0,00 & 0 & 61 & 61 \\
\hline$c^{\prime} \hat{E} T R E$ & 0,00 & 0 & 156 & 156 \\
\hline je sais pas & 1,75 & 1 & 56 & 57 \\
\hline Autres constructions & 9,69 & 111 & 1034 & 1145 \\
\hline GLOBAL & 7,83 & 112 & 1319 & 1431 \\
\hline
\end{tabular}

$\chi^{2}=27,7 ; \mathrm{p}=.000000014$

Les résultats de l'analyse des données restantes $(\mathrm{N}=1$ 145), présentés dans le Tableau 5, montrent que l'emploi de la particule demeure en-deçà des $10 \%$ (input = 0,082) et est sujet à un fort effet idiolectal. Alors que quatre locuteurs n'emploient jamais la particule pendant leur entrevue $(\mathrm{N}=184)$, se conformant à l'usage quasi-catégorique documenté pour les vernaculaires, un petit groupe se démarque dans la direction opposée par un emploi particulièrement élevé de ne. L'analyse à régression multiple, effectuée sur GoldVarb (Robinson, Lawrence et Tagliamonte 2001), révèle qu'aucun facteur social (sexe, âge ou catégorie professionnelle) ne réussit à lui seul à prédire l'usage de la particule négative. 
Toutefois, une analyse statistique tenant compte de la variation idiolectale montre que les locuteurs employant la particule se regroupent en trois grandes catégories: les grands utilisateurs et les utilisateurs modestes, qui favorisent ne mais l'emploient à des fréquences variables, et les faibles utilisateurs, qui défavorisent son emploi. On remarque également que la plupart des artistes (p. ex., chanteurs, comédiens, humoristes), faibles utilisateurs de ne, se conforment davantage à l'usage familier alors que trois des quatre animateurs de radio ou de télévision (Gilles P., Gaston L., Geneviève B.), favorisent un usage plus normatif, aligné sur la norme écrite. Cette différence pourrait être examinée davantage par le biais d'une analyse du positionnement des locuteurs au sein de leur communauté de pratique professionnelle (Eckert 2000).

Tableau 5: Emploi de ne et variation idiolectale dans le corpus Un Train ${ }^{6}$

\begin{tabular}{|c|c|c|c|c|c|c|c|}
\hline \multicolumn{8}{|l|}{ Tendance globale $=\mathbf{0 , 0 8 2}$} \\
\hline Groupe & Poids ${ }^{1}$ & $\%$ ne & $\mathbf{N}$ & Locuteurs & Poids & $\%$ ne & $\mathbf{N}$ \\
\hline \multirow[t]{4}{*}{ Grands utilisateurs } &, 879 & 40,2 & 127 & Gilles P. & ,922 & 51,5 & 33 \\
\hline & & & & Lise W. & ,899 & 44,4 & 18 \\
\hline & & & & André B. &, 867 & 36,7 & 49 \\
\hline & & & & Laurence J. &, 825 & 29,6 & 27 \\
\hline \multirow[t]{4}{*}{ Utilisateurs modestes } & ,669 & 15,7 & 172 & Andrée R. &, 745 & 20,7 & 58 \\
\hline & & & & Liza F. & ,681 & 16,0 & 50 \\
\hline & & & & Gaston L. & ,607 & 12,1 & 33 \\
\hline & & & & Geneviève B. &, 545 & 9,7 & 31 \\
\hline \multirow[t]{12}{*}{ Faibles utilisateurs } & ,363 & 5,0 & 662 & France C. & ,477 & 7,5 & 53 \\
\hline & & & & Brigitte P. &, 476 & 7,5 & 40 \\
\hline & & & & Dan B. &, 397 & 5,6 & 54 \\
\hline & & & & Ginette R. &, 392 & 5,5 & 55 \\
\hline & & & & Denis B. &, 388 & 5,4 & 56 \\
\hline & & & & Christian T. &, 377 & 5,1 & 78 \\
\hline & & & & Mario P. &, 365 & 4,9 & 41 \\
\hline & & & & Chantal P. &, 348 & 4,5 & 44 \\
\hline & & & & Ricardo L. &, 344 & 4,5 & 67 \\
\hline & & & & Éric L. &, 314 & 3,9 & 51 \\
\hline & & & & Isabel R. &, 301 & 3,7 & 54 \\
\hline & & & & Sylvie F. &, 251 & 2,9 & 69 \\
\hline écart & 516 & & & & 671 & & \\
\hline
\end{tabular}

Absence catégorique de ne: Mario T. (N=42), Lise D. (N=55), Guy L. (N=30) et Mario D. ( $\mathrm{N}=57)$

\subsubsection{La négation verbale et la variation stylistique}

Une comparaison des corpus révèle que l'emploi de la particule ne chez les cinq locuteurs interviewés dans les deux contextes passe de $12,1 \%(\mathrm{~N}=265)$ dans les entrevues Un Train à 21,8 \% (N=87) dans

6. Plus le poids relatif se rapproche de 1,000, plus la variante à l'étude est favorisée, et inversement pour un poids se rapprochant de, 000 . 
les entrevues Le Point, une augmentation statistiquement significative pour la cohorte ( $\mathrm{p}=0,034)$. Par contre, en examinant la variation individuelle (voir le Tableau 6), on note que cette augmentation est causée par deux politiciens ${ }^{7}$, Liza F. et Mario D., pour qui la particule négative semble servir de marque de formalité dans le corpus Le Point. L’usage des trois autres locuteurs - deux faibles utilisateurs et un grand utilisateur selon les données de Un Train-demeure constant entre les deux contextes d'entrevue.

Tableau 6: Emploi de ne dans deux contextes d'entrevue: Un Train et Le Point

\begin{tabular}{|c|c|c|c|c|c|c|}
\hline \multirow[t]{2}{*}{ Catégorie } & \multirow[t]{2}{*}{ Locuteurs } & \multicolumn{2}{|c|}{ Un Train } & \multicolumn{2}{|c|}{ Le Point } & \multirow{2}{*}{$\begin{array}{c}\text { Fisher Exact } \\
\text { p }\end{array}$} \\
\hline & & $\%$ ne & $\mathbf{N}$ & $\% n e$ & $\mathbf{N}$ & \\
\hline \multirow[t]{2}{*}{ Artistes } & Ginette R. & 5,5 & 55 & 4,3 & 23 & 1,000 \\
\hline & Dan B. & 5,6 & 54 & 0,0 & 26 & 0,547 \\
\hline \multirow[t]{3}{*}{ Politiciens } & Liza F. & 16,0 & 50 & 60,0 & 15 & 0,002 \\
\hline & André B. & 36,7 & 49 & 66,7 & 9 & 0,142 \\
\hline & Mario D. & 0,0 & 57 & 21,4 & 14 & 0,006 \\
\hline
\end{tabular}

Somme toute, nos données confirment les résultats d'études synchroniques et diachroniques basées sur les vernaculaires: la particule négative est essentiellement disparue du FQ oral, mais elle demeure accessible comme ressource linguistique servant à marquer la formalité associée au français «standard» écrit.

\section{Conclusion}

Par l'analyse d'un nouveau corpus d'entrevues télévisées à Radio-Canada et du corpus Le Point (Bigot 2008), nous avons démontré que l'usage linguistique des élites culturelles québécoises dans deux contextes d'entrevue représente assez fidèlement une norme objective du FQ oral soutenu, située à mi-chemin entre le vernaculaire largement décrit dans la littérature et l'utopique réalisation orale d'un 'standard' écrit. D'une part, l'emploi de l'auxiliaire avoir dans les contextes où l'écrit prescrit être est quasi-inexistant dans les deux corpus, ce qui semble indiquer une standardisation de cette variable, conformément au changement décrit dans les corpus de Montréal 1971-1995 (Sankoff et al 2004). D'autre part, l'emploi de la particule négative ne, dont l'emploi s'impose à l'écrit soutenu, ne dépasse le seuil des $20 \%$ que chez une minorité des 24 locuteurs, même s'il est largement supérieur au taux de moins de 1 \% recensé dans les vernaculaires (Sankoff et Vincent 1977, Poplack et St-Amand 2007). Est-ce dire que, même en contexte oral relativement surveillé, les Québécois n'auraient que peu recours à cette ressource stylistique? Une analyse des thèmes discursifs favorisant l'emploi de la particule négative dans notre corpus et un examen de la cooccurrence de la particule négative avec d'autres formes soutenues (p. ex., les pronoms relatifs complexes) nous permettra sans doute de déterminer si ne fait toujours partie de la grammaire de l'oral québécois spontané ou si, comme le suggère le modèle mixte

7. Étant donnée la petite taille du corpus, nous évitons d'interpréter trop hâtivement la variation stylistique propre aux politiciens. Il n'en demeure pas moins que le public québécois associe à «la plupart des politiciens du Québec» un usage linguistique particulier, qui se distinguerait autant de celui du «monde ordinaire qu'on voit dans les jeux télévisés» que de celui des "personnes qui lisent les nouvelles à Radio-Canada» (Maurais et Bouchard 1999). 
de la variation proposé par Villeneuve et Auger (2013), les locuteurs se trouvant en situation plus formelle puisent plutôt dans l'inventaire des formes associées à l'écrit pour dénoter le prestige à l'oral. Le fait que, pour les deux variables étudiées ici, certains locuteurs adoptent un usage catégorique de la variante familière ou soutenue pourrait indiquer que ces variables morphosyntaxiques ne sont pas des choix idéaux pour l'étude de la variation en français soutenu: ni l'alternance des auxiliaires, ni la négation verbale ne semble respecter le critère d'une vaste distribution sociale des formes au sein de la communauté (Coveney 1996/2002, Armstrong 2001). Nous espérons que l'analyse de variables morphosyntaxiques supplémentaires, telles que la référence temporelle au futur et les expressions de nécessité, nous permettra de mieux cerner les patrons de variation stylistique en FQ soutenu.

Somme toute, si l'école est appelée à «jouer un rôle décisif sur le plan de la compréhension des niveaux de langue» (Melançon 2015: 65), les éléments descriptifs que fournissent les corpus d'entrevues télévisuelles Un Train et Le Point permettent d'alimenter la discussion entourant l'oral en didactique du français. Par exemple, des ressources pédagogiques pourraient être développées à partir de la description nuancée d'une norme objective dominante en FQ afin de favoriser le développement d'une meilleure conscience sociolinguistique du continuum familier-soutenu chez les enseignants et les étudiants. Le fait qu'une partie du corpus Un Train soit disponible en coffret DVD facilite par ailleurs son utilisation éventuelle en milieu scolaire. Enfin, des chercheurs américains s'appuient depuis déjà près de quarante ans sur la recherche en sociolinguistique pour atténuer les inégalités sociales et la discrimination basée sur la langue, notamment en aidant les enseignants et les élèves à mieux comprendre les distinctions entre l'anglais normatif et diverses variétés vernaculaires (Charity Hudley et Mallinson 2011, 2014, Rickford 1999). Au Canada, une meilleure compréhension de la variation sociolinguistique et une conception plus objective de la notion de norme(s) pourrait donc permettre d'enrichir et, surtout, de nuancer les discours sur les français d'ici.

\section{Références bibliographiques}

Armstrong, N. 2001. Social and stylistic variation in spoken French: a comparative approach. Amsterdam; Philadelphia: John Benjamins.

Ashby, W. J. 1984. "The elision of /1/ in French clitic pronouns and articles". Dans E. Pulgram (dir.), Romanitas: Studies in Romance Linguistics. Ann Arbor: University of Michigan Press: 1-16.

Ashby, W. J. 2001. "Un nouveau regard sur la chute du ne en français parlé tourangeau: S'agit-il d'un changement en cours». Journal of French Language Studies 11 (1): 1-22. doi: http://dx.doi.org/10.1017/ S0959269501000114

Association québécoise des professeurs de français [AQPF]. 1977. «Le discours de M. Jacques-Yvan Morin: L'enseignement du français ». Québec français (28) : 13-15.

Bachy, S., Dister, A., Francard, M., Géron, G., Giroul, V., Hambye, P., Simon, A. C. et Wilmet, R. 2004/2007. Conventions de transcription régissant les corpus de la banque de données VALIBEL.

Barbaud, P. 1998. «Dissidence du français québécois et évolution dialectale». Revue québécoise de linguistique 26 (2) : 107-128. doi : http://dx.doi.org/10.7202/603156ar

Bauche, H. 1920/1946. Le langage populaire: Grammaire, syntaxe et dictionnaire du français tel qu'on le parle dans le peuple de Paris, avec tous les termes d'argot usuel. Paris: Payot.

Beeching, K. 2012. "Sociolinguistic aspects of lexical variation in French". Dans T. Pooley et D. Lagorgette (dir.), On linguistic change in French: Socio-historical approaches / Le changement linguistique en francais: Aspects socio-historiques. Chambéry: Presses universitaires de Savoie: 37-54.

Bell, A. 1984. "Language style as audience design". Language in Society 13 (2): 145-204. doi: http://dx.doi. org/10.1017/S004740450001037X 
Bell, A. 2001. "Back in style: Reworking audience design”. Dans P. Eckert et J. R. Rickford (dir.), Style and sociolinguistic variation. Cambridge: Cambridge University Press: 139-169.

Bigot, D. 2008. "Le Point» sur la norme grammaticale du français québécois oral. Thèse de doctorat, Université du Québec à Montréal, Montréal.

Bigot, D. 2010. «La norme grammaticale du français québécois oral: Des questions, une réponse». Dans F. Martineau (dir.), Vues sur les français d'ici. Québec Presses de l'Université Laval: 9-30.

Bigot, D. 2011. «De la norme grammaticale du français parlé au Québec». Arborescences 1: 1-18. doi : http:// dx.doi.org/10.7202/1001939ar.

Bigot, D. et Papen, R. A. 2013. "Sur la «norme» du français oral au Québec (et au Canada en général)». Langage et société 146: 115-132. doi : http://dx.doi.org/10.3917/1s.146.0115.

Blondeau, H. 2001. "Real-time changes in the paradigm of personal pronouns in Montreal French". Journal of Sociolinguistics 5 (4): 453-474. doi: http://dx.doi.org/10.1111/1467-9481.00160.

Blondeau, H. 2006. "La trajectoire de l'emploi du futur chez une cohorte de Montréalais francophones entre 1971 et 1995». Revue canadienne de linguistique appliquée 9 (2): 73-98.doi: http://dx.doi. org/10.7202/015840ar.

Blondeau, H., Sankoff, G. et Charity, A. 2002. «Parcours individuels dans deux changements linguistiques en cours en français montréalais». Revue québécoise de linguistique 31 (1): 13-38. doi: http://dx.doi. org/10.7202/006843ar.

Blondeau, H., Tremblay, M. et Drouin, P. 2014. «Hybridité et variation dans les SMS : Le corpus Texto4Science et l'oralité en français montréalais». Canadian Journal of Linguistics / Revue canadienne de linguistique 59 (1) : 137-165. doi : http://dx.doi.org/10.1017/S0008413100000189.

Bouchard, P. et Maurais, J. 2001. «Norme et médias. Les opinions de la population québécoise». Terminogramme 97-98: 111-126.

Bourhis, R. Y. et Landry, R. 2002. "La loi 101 et l'aménagement du paysage linguistique au Québec». Revue d'aménagement linguistique 2002: 107-132.

Cajolet-Laganière, H. et Martel, P. 1995. La qualité de la langue au Québec (Vol. 18). Québec: Institut québécois de recherche sur la culture.

Cedergren, H. J. et Sankoff, D. 1974. "Variable rules: Performance as a statistical reflection of competence". Language 50 (2): 333-355.

Charity Hudley, A. H. et Mallinson, C. 2011. Understanding English language variation in U.S. schools. New York: Teachers College Press.

Charity Hudley, A. H. et Mallinson, C. 2014. We do language: English language variation in the secondary English classroom. New York: Teachers College Press.

Conwell, M. J. et Juilland, A. G. 1963. Louisiana French grammar. The Hague: Mouton.

Coupland, N. 2007. Style: Language variation and identity. Cambridge University Press.

Coveney, A. 1996/2002. Variability in spoken French: A sociolinguistic study of interrogation and negation. Bristol: Elm Bank Publications.

Cox, T. B. 1998. "Vers une norme pour un cours de phonétique française au Canada». Canadian Modern Language Review / Revue canadienne des langues vivantes 54 (2): 172-197. doi : http://dx.doi.org/10.3138/ cmlr.54.2.172.

Deshaies, D. 1981. Le français parlé dans la ville de Québec: une étude sociolinguistique. Québec: Centre international de recherche sur le bilinguisme.

Dewaele, J.-M. 2004. "The acquisition of sociolinguistic competence in French as a foreign language: An overview”. Journal of French Language Studies 14 (3): 301-319. doi: http://dx.doi.org/10.1017/ S0959269504001814.

Dubois, S. (dir.) 2011. Une histoire épistolaire de la Louisiane. Québec: Presses de l’Université Laval.

Eckert, P. 2000. Language variation as social practice: The linguistic construction of identity in Belten High. Malden, MA: Blackwell. 
François, D. 1974. Français parlé: Analyse des unités phoniques et significatives d'un corpus recueilli dans la région parisienne. Paris: Société d'études linguistiques et anthropologiques de France.

Frei, H. 1929/1971. La grammaire des fautes. Geneva: Slatkine Reprints.

French, L. M. et Beaulieu, S. 2016. "Effects of sociolinguistic awareness on French L2 learners' planned and unplanned oral production of stylistic variation". Language Awareness 25 (1-2): 1-17. doi : http://dx.doi. org/10.1080/09658416.2015.1122024.

Gadet, F. 1995. «Norme, variation, évaluation». Histoire Épistémologie Langage 11 (1) : 18-22.

Gadet, F. 2000. «Des corpus pour ne...pas». Dans M. Bilger (dir.), Corpus: méthodologie et applications linguistiques. Paris: Champion: 156-167.

Gendron, J.-D. 1990. «Modèles linguistiques, évolution sociale et normalisation du langage». Dans N. Corbett (dir.), Langue et identité. Le français et les francophones d'Amérique du Nord. Québec: Presses de l'Université Laval: 369-388.

Gesner, E. 1978. «L'emploi des auxiliaires avoir et être dans le parler de la Baie Sainte-Marie, Nouvelle-Ecosse». Dans G. W. Patterson (dir.), Papers from the Second Annual Meeting of the Atlantic Provinces Linguistic Association. Halifax: Mount Saint Vincent University: 16-22.

Grevisse, M. et Goosse, A. 1993. Le bon usage: Grammaire française (13e édition). Paris: Duculot.

Guillot, H. 1932. Ce qu’il faut dire - ce qu’il ne faut pas dire. Dédié à la jeunesse alsacienne si désireuse de parler correctement la langue française ( $4^{\mathrm{e}}$ édition). Strasbourg: Dernières Nouvelles.

Hallion Bres, S. 2004. "Quelques aspects de la morphosyntaxe du verbe en franco-manitobain ». Cahiers francocanadiens de l'Ouest 16 (1-2) : 79-97.

Hallion Bres, S. 2006. «Similarités morphosyntaxiques des parlers français de l'Ouest canadien». Revue de l'Université de Moncton 37 (2): 111-131. doi: http://dx.doi.org/10.7202/015842ar.

Hamel, M. 1995. «Les faits et les théories qui soutiennent les interventions éducatives au préscolaire». Dans N. Royer (dir.), Éducation et intervention au préscolaire. Montréal: Gaëtan Morin : 29-54.

King, R. et Nadasdi, T. 2000. "How auxiliaries be/have in Acadian French". Dans P. Balcom, L. Beaulieu et G. 1. Chevalier (dir.), Papers from the 24th Annual Meeting of the Atlantic Provinces Linguistic Association. Moncton: Université de Moncton: 61-72.

Labov, W. 1972. Sociolinguistic patterns. Philadelphia: University of Pennsylvania Press.

Labov, W. 1984. "Field methods of the project on language change and variation". Dans J. Baugh et J. Sherzer (dir.), Language in use: Readings in sociolinguistics. Englewood Cliffs, NJ: Prentice Hall: 28-53.

Labov, W. 2001. "The anatomy of style-shifting”. Dans P. Eckert et J. R. Rickford (dir.), Style and sociolinguistic variation. Cambridge: Cambridge University Pres: 85-108.

Léard, J.-M. 1995. Grammaire québécoise d'aujourd'hui: comprendre les québécismes. Montréal: Guérin universitaire.

Lemelin, A. 1979). «Passe-Partout: la prématernelle des parents». Éducation Québec 9: 21-22.

Martel, P. et Cajolet-Laganière, H. 2000. «Le français au Québec: un standard à décrire et des usages à hiérarchiser ». Dans M. Plourde et al. (dir.), Le français au Québec. 400 ans d'histoire et de vie. Montréal: Fides; Les Publications du Québec: 379-391.

Martineau, F. 2005. «Perspectives sur le changement linguistique: Aux sources du français canadien». Canadian Journal of Linguistics / Revue canadienne de linguistique 50: 173-213.

Martineau, F. et Mougeon, R. 2003. "A sociolinguistic study of the origins of ne deletion in European and Quebec French”. Language 79 (1): 118-152. doi : http://dx.doi.org/10.1353/lan.2003.0090.

Melançon, B. 2015. Le niveau baisse! (et autres idées reçues sur la langue). Montréal: Del Busso éditeur.

Meney, L. 2017. Le français québécois entre réalité et idéologie: Un autre regard sur la langue. Étude sociolinguistique. Québec: Presses de l'Université Laval.

Moreau, M.-L. 1986. «Les séquences préformées: entre les combinaisons libres et les idiomatismes. Le cas de la négation avec ou sans ne». Le français moderne 54: 137-160.

Moreau, M.-L. 1997. Sociolinguistique: Les concepts de base. Liège: Mardaga. 
Mougeon, R. et Beniak, É. 1989. "Language contraction and linguistic change: The case of Welland French". Dans N. Dorian (dir.), Investigating obsolescence. Cambridge: Cambridge University Press: 287-312.

Mougeon, R. et Beniak, É. 1991. Linguistic consequences of language contact and restriction: the case of French in Ontario, Canada. Oxford; New York: Oxford University Press.

Mougeon, R., Nadasdi, T. et Rehner, K. 2010. The sociolinguistic competence of immersion students. Bristol; Buffalo: Multilingual Matters.

Nadasdi, T., Mougeon, R. et Rehner, K. 2005. "Learning to speak everyday (Canadian) French". Canadian Modern Language Review / Revue canadienne des langues vivantes 61 (4): 543-561. doi: http://dx.doi. org/10.3138/cmlr.61.4.543.

Ostiguy, L. et Tousignant, C. 2008. Les prononciations du français québécois: normes et usages (2 édition). Montréal: Guérin universitaire.

Paradis, C. 1985. An acoustic study of variation and change in the vowel system of Chicoutimi and Jonquiere (Québec). Thèse de doctorat, University of Pennsylvania.

Péronnet, L. 1991. «Système des modalités verbales dans le parler acadien du sud-est du Nouveau-Brunswick». Journal of the Atlantic Provinces Linguistic Association 13: 85-98.

Poplack, S. 1989. "The care and handling of a megacorpus: The Ottawa-Hull French project”. Dans R. Fasold et D. Schiffrin (dir.), Language change and variation. Amsterdam; Philadelphia: John Benjamins: 411-451.

Poplack, S. et St-Amand, A. 2007. "A real-time window on 19th-century vernacular French: The Récits du français québécois d'autrefois». Language in Society 36: 707-734. doi: http://dx.doi.org/10.1017/ S0047404507070662.

Poplack, S. et St-Amand, A. 2009. "Les Récits du français québécois d'autrefois: Reflet du parler vernaculaire du $19^{\mathrm{e}}$ siècle». Canadian Journal of Linguistics / Revue canadienne de linguistique 54 (3) : 511-546. doi : http:// dx.doi.org/10.1353/cjl.0.0060.

Radio-Canada. 2003. Un français de qualité: Une priorité pour Radio-Canada. Rapport du Groupe de travail sur la qualité de la langue (GTQL) présidé par monsieur Yanick Villedieu.

Rea, B. 2015. « «Je m’ai fait mal quand j’ai tombé»: Questions d'auxiliarité et de réflexivité dans le français parlé à Montréal (1971-2013)». ScriptUM: la revue du colloque VocUM 1:3-18.

Reinke, K. 2005. La langue à la télévision québécoise: aspects sociophonétiques. Gouvernement du Québec, Office québécois de la langue française.

Remysen, W. 2011. "Les représentations identitaires dans le discours normatif des chroniqueurs de langage canadiens-français depuis le milieu du XIx siècle». Journal of French Language Studies 22 (3) : 1-26. doi: http://dx.doi.org/10.1017/S0959269511000536.

Rickford, J. R. 1999. "Using the vernacular to teach the standard". Dans J. R. Rickford (dir.), African American Vernacular English. Malden, MA: Blackwell: 329-347.

Robinson, J., Lawrence, H. et Tagliamonte, S. A. 2001. Goldvarb 2001 : A multivariate analysis application for Windows. York: University of York, Department of Language and Linguistic Science.

Russo, M. et Roberts, J. 1999. "Linguistic change in endangered dialects: The case of alternation between avoir and être in Vermont French". Language Variation and Change 11 (1): 67-86.

Sankoff, D., Sankoff, G., Laberge, S. et Topham, M. 1976. "Méthodes d'échantillonnage et utilisation de l'ordinateur dans l'étude de la variation grammaticale». Cahiers de Linguistique de l'Université du Québec $6: 85-125$.

Sankoff, G. et Blondeau, H. 2007. "Language change across the lifespan: /r/ in Montreal French". Language 83 (3) : 560-588. doi : http://dx.doi.org/10.1353/lan.2007.0106.

Sankoff, G. et Thibault, P. 1977. «L'alternance entre les auxiliaires avoir et être en français parlé à Montréal». Language française 34: 81-108.

Sankoff, G. et Thibault, P. 1980. "The alternation between the auxiliaries avoir and être in Montreal French". Dans G. Sankoff (dir.), The social life of language. Philadelphia: University of Pennsylvania Press: 311-345. 
Sankoff, G., Thibault, P. et Wagner, S. 2004. An apparent time paradox: change in Montreal French auxiliary selection, 1971-1995. Communication présentée à NWAV 33 (33rd New Ways of Analyzing Variation), Ann Arbor, MI.

Sankoff, G. et Vincent, D. 1977. «L'emploi productif du ne dans le français parlé à Montréal». Le français moderne 45 : 243-256.

Tagliamonte, S. A. 2006. Analysing sociolinguistic variation. Cambridge: Cambridge University Press.

Thibault, P. et Vincent, D. 1990. Un corpus de français parlé: Montréal 84, historique, méthodes et perspectives de recherche Québec: Université Laval.

Villeneuve, A.-J. et Auger, J. 2013. “'Chtileu qu'i m’freumereu m’bouque i n'est point coér au monne': Grammatical variation and diglossia in Picardie”. Journal of French Language Studies 23 (1) : 109-133. doi: http://dx.doi.org/10.1017/S0959269512000385.

Vincent, D., Laforest, M. et Martel, G. 1995. «Le corpus de Montréal 1995: Adaptation de la méthode d'enquête sociolinguistique pour l'analyse conversationnelle». Dialangue 6:29-46.

Willis, L. 2000. Ettre ou ne plus être: Auxiliary alternation in Ottawa-Hull French. Mémoire de maittrise, Université d'Ottawa. 